\title{
Neuartige Diamant-Metall-Verbundfolien zum Feinschleifen von Saphir
}

\author{
Michael Gruner, Marcel Mück, Asta Richter
}

\section{Zusammenfassung}

Das ProInno-Projekt „Feinschleifen von Saphir“ (www.tfhwildau.de/mmueck) beschäftigt sich mit der Weiterentwicklung eines Verbundwerkstoffes für den Feinschleifprozess. Bei diesem Verbundwerkstoff handelt es sich um Diamantkörner, die in einer Kupfer-Zinn-Matrix eingebettet sind. In dem Projekt werden die Wirkungsmechanismen des neuartigen Verbundmaterials genauer untersucht. Mit diesen Erkenntnissen erfolgt eine Optimierung der einzelnen Komponenten und des Mischungsverhältnisses auf praxisbezogene Eigenschaften des Werkstoffes. Es ist geplant, hieraus ein marktfähiges Produkt zur Oberflächenbearbeitung harter Materialien zu entwickeln.

Der Werkstoff wurde auf Morphologie und Verteilung der einzelnen Komponenten untersucht. Es gibt eine Verdichtung des Gefuiges im Randbereich von ca. 30 $\mu \mathrm{m}$ durch den Walzprozess. Es wurden Bronzen mit $3 \%$ und $5 \%$ Zinnanteil hergestellt. Die Nanoindentationsmessungen konnten keine klare Abhängigkeit der plastischen und elastischen Eigenschaften der Bronze vom Zinnanteil aufzeigen. Die in dem Werkstoff enthaltenen Diamanten sind nicht vollständig von der Bronze umschlossen, sondern werden von einzelnen Körnern in dem Gefuige gehalten. Es konnte gezeigt werden, dass die eingebetteten Diamanten keine wesentlichen Änderungen am Bronzegefuige verursachen. Die Diamanten erscheinen gleichmäßig verteilt, wobei es aber vereinzelt zu Diamantanhäufungen von zwei bis drei Diamanten kommen kann.

Ein erster Tribologietest zeigt eine Abhängigkeit des Abriebverhaltens des Werkstoffes vom Diamantanteil. Es konnte beispielhaft gezeigt werden, dass ein höherer Diamantanteil zu einem geringeren Verschleiß der Verbundfolie beiträgt.

\section{Einleitung}

Synthetisch hergestellter Saphir [1] gewinnt aufgrund seiner exzellenten optischen, mechanischen, thermischen und chemischen Materialeigenschaften immer größere Bedeutung in Hightech-Branchen, wie der Mikroelektronik, Medizintechnik, Optik und Nanotechnik. Dabei spielt die Oberflächenqualität des harten Materials Saphir eine entscheidende Rolle.

Im Rahmen des Prolnno-Projektes „Feinschleifen von Saphir" wird gemeinsam mit der Vollstädt Diamant GmbH in Seddin eine neue Werkzeugtechnologie zur Herstellung von Diamant-Metall-Verbundfolien entwickelt. Feine synthetische Diamantkörner unterschiedlicher Körnung werden in einer festen Metallmatrix eingelagert, so dass ein Pad entsteht, welches beim Schleifprozess flexibel eingesetzt werden kann. Die Diamant-Verbundfolien werden bezuiglich ihrer Wirkungsmechanismen und ihrer Eigenschaften charakterisiert und geprüft. Dabei kommen die moderne Lichtmikroskopie, Rastersondenverfahren, tribologische Untersuchungen und nanometrisch-mechanische Eigenschaftstests (Härte, E-Modul) zum Einsatz. Die entwickelten Qualitätskriterien tragen zu einem hohen Standard bei der Herstellung des Diamant-Metall-Verbundwerkstoffes bei. Mit dem neuartigen Diamantwerkzeug ist es möglich, Oberflächen harter Stoffe im Feinschleifprozess zu bearbeiten.

Dieses Verfahren hat gegenuiber den klassischen Schleifund Läppprozessen viele Vorteile. Beim konventionellen Läppen wird eine Suspension versetzt mit Läppkörnern benutzt, die dem Prozess kontinuierlich zugefuihrt wird. Diese Läppkörner aus Siliziumkarbid, Borkarbid, Aluminiumoxid oder auch Diamant zersplittern schon nach kurzer Einsatzzeit und reduzieren damit den Abtrag deutlich. Eine kontinuierliche Entsorgung der Suspension aus der Maschine ist deshalb erforderlich. Derzeit werden jährlich ungefähr 5.000 t bis 10.000 t Läppsuspension in der Bundesrepublik Deutschland verbraucht, die häufig als Sondermüll entsorgt werden müssen. [2] Außer den hohen Entsorgungskosten stellen die geringen Abtragsraten, der hohe Aufwand furr die Reinigung der Werkstuicke und die schlechte Automatisierbarkeit des Fertigungsprozesses die gravierendsten Nachteile des Läppens dar. Der Einsatz von gebundenem Diamant fuihrt zu einer deutlichen Reduzierung der Entsorgungskosten und zu höheren Abtragsraten. Die geringere Verschmutzung der Werkstücke bedeutet einen kleineren Reinigungsaufwand und eröffnet das Potenzial für eine Automatisierung des Schleifprozesses.

\section{Herstellung der Diamant-Metall- Verbundfolien}

Die Herstellung der Diamant-Metall-Verbundwerkstoffe für das Feinschleifen erstreckt sich über eine Reihe von einzelnen Arbeitsgängen. [3] Ausgangsstoffe sind Metallpulver aus Kupfer und Zinn und industriell gefertigte Diamanten. Ein wesentlicher Bestandteil ist dendritisches (griech. dendron, Baum) Kupfer. Dieses eignet sich gut zum Herstellen von Folien, da sich die einzelnen „Äste“ miteinander verhaken können und damit eine stabile 


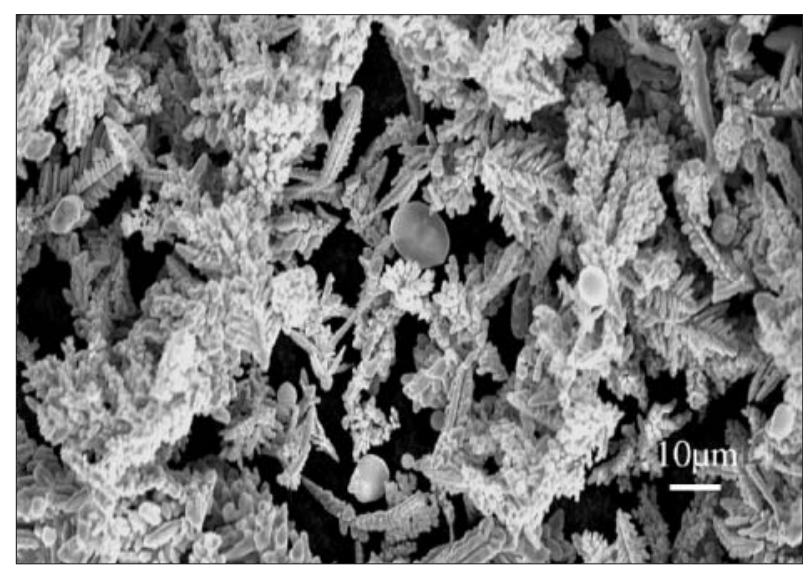

Abb. 1: REM-Aufnahme des Gemisches mit den Ausgangspulvern aus dendritischem Kupfer und kugelförmigem Zinn

Verbindung ergeben. Abbildung 1 zeigt eine RasterElektronen-Mikroskopieaufnahme (REM) des verwendeten Kupfer-Zinn-Gemisches als Pulver.

Im ersten Arbeitsgang wird eine Mischung aus den Metallpulvern Kupfer und Zinn und den synthetischen Diamanten hergestellt. Diese wird durch ein mehrmaliges Durchfuihren eines Sieb-Mischverfahrens homogenisiert. Der Zinnanteil kann zwischen $3 \%$ bis maximal $10 \%$ liegen und ist maßgeblich für die Festigkeit der entstehenden Bronze verantwortlich. Mit steigendem Zinngehalt wird die Folie fester aber auch spröder. Mittels Variation dieses Parameters muss ein Kompromiss zwischen Festigkeit und flexibler Verformbarkeit der Metallfolien gefunden werden. Um die Einbindung der Diamanten in die Bronze zu unterstuitzen, wird das Diamantpulver angefeuchtet. Der Anteil der Diamanten liegt bei 8 bis $25 \%$ der Gesamtmasse, die Korngrößen variieren zwischen $10 \mu \mathrm{m}$ und $28 \mu \mathrm{m}$.

Zur Herstellung einer Rohfolie wird die Mischung durch Walzen bei einem Druck von 200 ... 300 GPa verdichtet. Das Pulver wird gleichmäßig, mittels eines nach unten offenen Vorratsbehälters, durch zwei langsam gegeneinander laufende Walzen mit definiertem Abstand gepresst. Bei diesem Prozess des mechanischen Legierens entsteht eine noch brüchige Rohfolie aus Bronze mit eingelagerten Diamanten, der so genannte Grünling. Je größer die Zinnund Diamantanteile sind, desto spröder ist die Verbundfolie. Bei einem hohen Grad an Versprödung kann es zum Abriss der Folie während des Walzvorganges kommen. Der Anteil des Zinns und der Diamanten muss also auch in Hinsicht auf den Walzprozess optimiert werden.

Nach der Fertigung des Grünlings wird dieser in einzelnen Arbeitsschritten gesintert und nachgewalzt. Das Sintern ist ein Schluisselprozess in der gesamten Technologie der Herstellung der Verbundfolien. Damit wird ein Fertigungsverfahren bezeichnet, in dessen Verlauf ein poröser, körniger Stoff aus verschiedenen Komponenten in einen dichten Festkörper überführt wird. Dabei nimmt die mechanische Festigkeit zu. Da die Porosität des Ausgangskörpers mit einer großen inneren Oberfläche verbunden ist, kann man die Triebkraft des Sintervorgangs in dem Bestreben des Systems zur Verminderung der Oberfläche sehen. [4] Gesintert wird unter einer Wasserstoffatmosphäre bei einer Temperatur von ca. $800{ }^{\circ} \mathrm{C}$. Nach dem Sintern werden die einzelnen
Folien auf ihre endgültige Materialstärke gewalzt. Dabei entstehen Verspannungen im Material, welche durch Nachsintern bei geringeren Temperaturen (ca. $500{ }^{\circ} \mathrm{C}$ ) aufgehoben werden. Damit ist der Herstellungsprozess für die Schleiffolien abgeschlossen. Nun können diese mittels Stanzen, Laser- oder Wasserstrahlschneiden in eine für den künftigen Anwendungszweck erforderliche Form gebracht werden. Eine Übersicht zu den beschriebenen Arbeitsgängen bietet Abbildung 2.

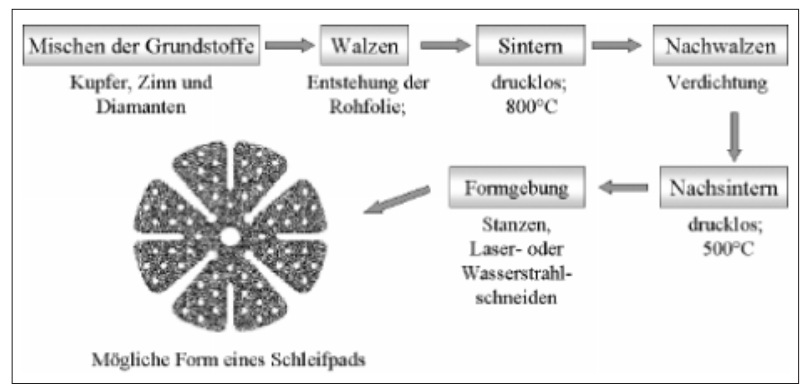

Abb. 2: Übersicht der Arbeitsgänge zur Herstellung der Schleiffolien

Die Abbildung 3 zeigt die Herstellungsparameter der verschiedenen Chargen hinsichtlich deren Zusammensetzung und der durchgefuihrten Verarbeitungsschritte.

\begin{tabular}{|c|c|c|c|c|c|c|c|c|c|}
\hline$\frac{\mathscr{0}}{0}$ & $\begin{array}{l}\tilde{N} \\
\frac{N}{N} \\
\vec{N}\end{array}$ & 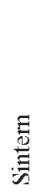 & 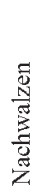 & 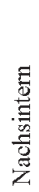 & 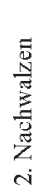 & 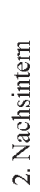 & 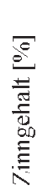 & 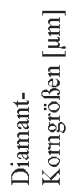 & 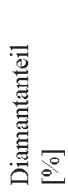 \\
\hline $\mathrm{C} 1$ & + & + & + & + & & & 3 & $28 / 20$ & 25 \\
\hline $\mathrm{C} 2$ & + & + & + & + & & & 5 & $28 / 20$ & 25 \\
\hline C3 & + & + & + & + & & & 3 & $20 / 14$ & 25 \\
\hline $\mathrm{C} 4$ & + & + & + & + & & & 5 & $20 / 14$ & 25 \\
\hline $\mathrm{C} 5$ & + & + & + & + & & & 5 & $14 / 10$ & 12 \\
\hline C6 & + & + & + & + & & & 5 & $14 / 10$ & 8 \\
\hline D3 & + & + & + & + & + & + & 3 & $20 / 14$ & 25 \\
\hline D4 & + & + & + & + & + & + & 5 & $20 / 14$ & 25 \\
\hline
\end{tabular}

Abb. 3: Überblick der Herstellungsparameter

\section{Analyse}

\subsection{Morphologie und Verteilung der Komponenten im Verbundwerkstoff}

Der Verteilung der Komponenten im Verbundwerkstoff wird eine besondere Aufmerksamkeit gewidmet, da sie von hoher Bedeutung für die Anwendung als Schleifwerkzeug ist. Sind die Diamanten nicht gleichmäßig verteilt und bilden Anhäufungen, können diese als Überkörner wirken und in der Oberfläche des zu bearbeitenden Materials Kratzer und Riefen erzeugen. Weist die Bronze starke lokale Unterschiede in ihren Eigenschaften auf, kann dies zu einer ungleichmäßigen Abnutzung des Werkzeuges fuihren. Ein schlechter Schliff sowie verkuirzte Standzeiten können daraus resultieren.

Eine quantitative Beurteilung der Homogenität der verschiedenen Eigenschaften ist aufwendig, da dies eine hohe Anzahl von Messungen voraussetzt. Eine erste Einschätzung erlauben jedoch bereits wenige Stichproben. Als Kriterien wurden die Gefuigestruktur, die Diamantverteilung und die Härte der Bronze betrachtet. 
Die Gefuigeuntersuchungen wurden an Querbrüichen der Verbundfolien mittels Raster-Elektronen-Mikroskopie (REM) durchgefuihrt. Das Gefuige erscheint gleichmäßig ungeordnet entlang der Längsseite der Proben. Eine querseitige Betrachtung zeigt Unterschiede in der Verdichtung des Gefuiges. In Abbildung 4 ist eine besonders starke Verdichtung in den ersten 30 Mikrometern des Randbereiches der Probe erkennbar. Dieser Effekt wird durch das Walzen hervorgerufen. Die Kraft wird besser in die oberflächennahen Gebiete übertragen als in das Innere der Folie.

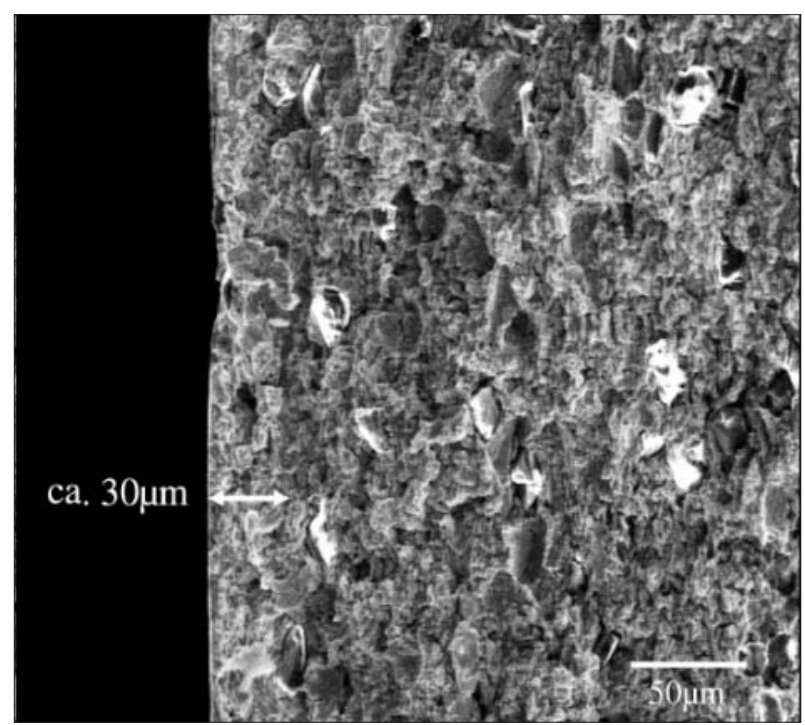

Abb. 4: REM-Aufnahme eines Querbruches der Diamant-Metall-Verbundfolien

In der Abbildung 4 sind auch einzelne Diamanten (helle Körner) zu erkennen, die sich mitunter aufgrund des hohen Diamantanteils von $25 \%$ in dieser Probe zum Teil dicht beieinander befinden, aber keine Cluster bilden. Dass solche Cluster in den Proben existieren, zeigt Abbildung 5, in der eine Ansammlung von drei direkt aneinander grenzenden Diamanten aufgezeigt wird. Die Diamanten sind in eine feinkörnige lamellenartige Bronzematrix eingebettet. Durch den Walzprozess wird eine Textur erzeugt, so dass die Kornstruktur länglich geformte Gebiete aufweist (Abb. 5).

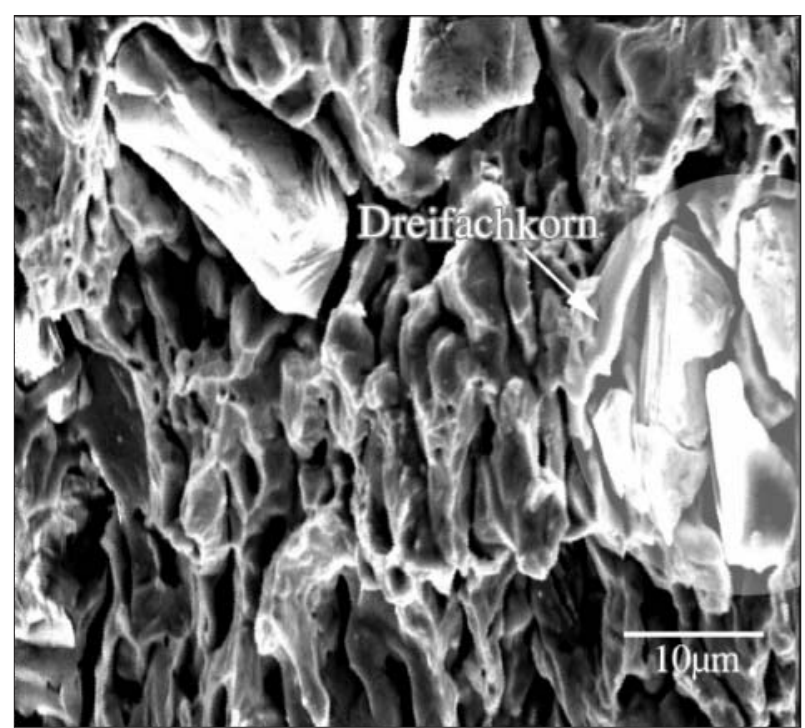

Abb. 5: REM Aufnahme der Diamant-Metall-Verbundfolien - mit Cluster von drei Diamanten
Als drittes Kriterium zur Einschätzung der Morphologie und Verteilung der Komponenten im Verbundwerkstoff wurden die mechanischen Eigenschaften der Bronzematrix herangezogen. Die mechanischen Kenngrößen wurden lokal mit dem Nanoindenter [5-7] bestimmt, welcher gleichzeitig als Raster-Kraft-Mikroskop (SFM) verwendet werden kann. Dies hat den Vorteil, dass die Härte und das E-Modul der Bronze durch sehr kleine gezielte Messeindrücke weitestgehend unabhängig von den eingelagerten Diamanten bestimmt werden konnten. Es wurde mit Lasten zwischen $1 \mathrm{mN}$ und $3 \mathrm{mN}$ gearbeitet. Abbildung 6 zeigt die Verteilung des Elastizitätsmoduls über 7 Messungen an 2 unterschiedlichen Gebieten auf der Probe. Jede Einzelmessung besteht aus mehreren Eindringzyklen im Multiindentverfahren. [8] Jeder Zyklus liefert einen Wert für das E-Modul, der hauptsächlich die Bronzematrix charakterisiert. Die Werte in Abbildung 6 stellen die Mittelwerte dieser Einzelzyklen dar, die Standardabweichung kann als Toleranzbereich der Einzelmessung betrachtet werden.

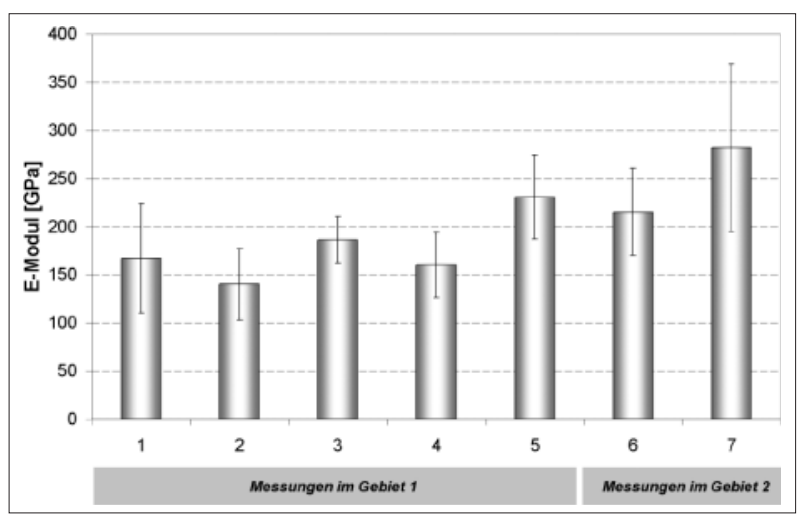

Abb. 6: E-Modul-Verteilung der Bronzematrix des Verbundwerkstoffes

Die Messreihe lässt eine starke Schwankung des E-Moduls erkennen. Die Werte variieren zwischen $140 \mathrm{GPa}$ und 280 GPa und schwanken auch innerhalb eines Messgebietes um ca. $100 \mathrm{GPa}$. Dies lässt auf einen inhomogenen Werkstoff schließen. Die örtliche Auflösung dieser Messung liegt im Submikrometerbereich. Es werden also die mechanischen Eigenschaften einzelner Gefuigekörner der Metallmatrix bestimmt. Die Messung kann durch benachbarte Poren beeinflusst werden. Diese Inhomogenität im Submikrometer- und Mikrometerbereich ist dem Herstellungsverfahren dieses Hartstoff-Metall-Verbundmaterials geschuldet und stellt keine Beurteilung der gleichmäßigen Verteilung von praktisch relevanten mechanischen Eigenschaften des Werkstoffes dar.

\subsection{Einbettung der Diamanten}

Eine erste Beurteilung der Diamanteinbettung kann anhand der REM-Aufnahmen vollzogen werden. In Abbildung 5 ist zu erkennen, dass die Diamanten nicht vollständig von der Bronze umschlossen sind, sondern von einzelnen anliegenden Körnern und Poren umgeben sind.

Um eventuelle Änderungen der Bronze im Bereich der Diamanten zu erkennen, wurden Eindringversuche in der Bronze mit verschiedenen Abständen zu den Diamanten durchgefuihrt. Beispiele solcher Indentreihen sind in Abbildung 7 dargestellt. 


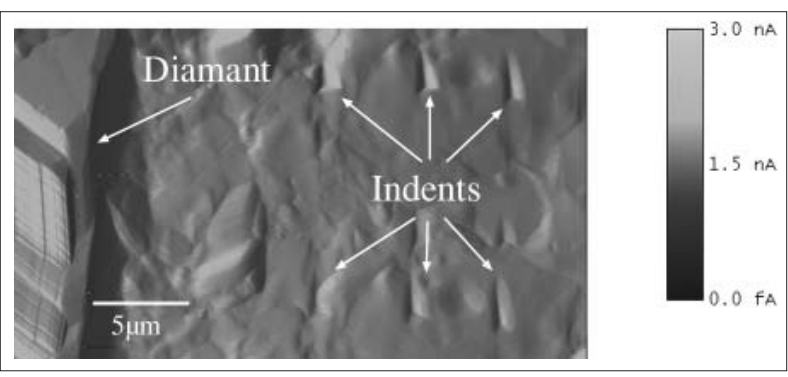

Abb. 7: SFM Aufnahme an der Diamant-Metall-Verbundfolie mit Indentreihen in Richtung des eingebetteten Diamanten

Die Messungen wurden für mehrere Proben durchgefuihrt. Abbildung 8 zeigt eine Zusammenfassung der Ergebnisse. Das gemessene E-Modul der Bronzen schwankt zwischen $100 \mathrm{GPa}$ und $200 \mathrm{GPa}$. Nur in unmittelbarer Nähe der Diamanten (Abstand $\leq 3 \mu \mathrm{m}$ ) werden Werte bis zu 360 GPa gemessen. Dies spricht gegen eine Beeinflussung der Gefuigestruktur der Bronzen durch die Diamanten. Bei Eindringversuchen wirkt nicht nur das Material, das sich direkt unter dem Prüfkörper befindet, auf die Messung ein. Alles Material, das sich innerhalb einer Halbkugel mit einem ungefähren Radius von dem dreifachen des Indentdurchmessers befindet, beeinflusst das Verfahren. [5] Die Eindrücke der Nanohärtemessung sind ungefähr $1 \mu \mathrm{m}$ breit, daher sind die hohen Werte in den ersten drei Mikrometern des Diagramms auf eine direkte Beeinflussung der Messung durch die Diamanten zuruickzuführen.

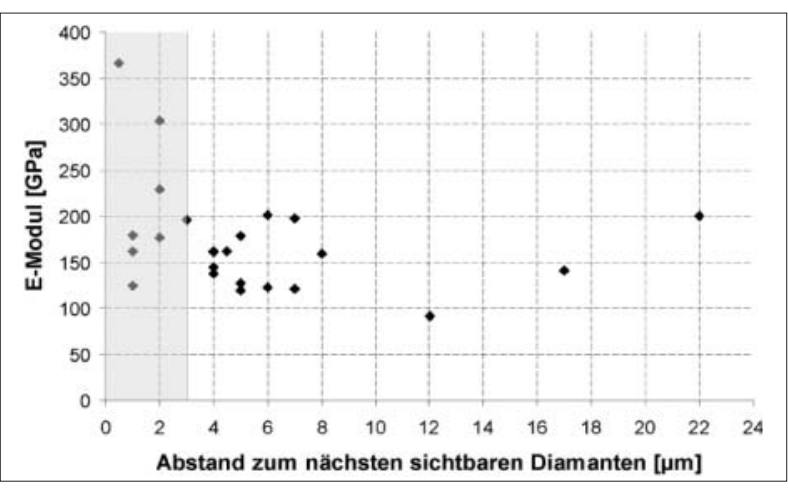

Abb. 8: Einfluss der eingebetteten Diamanten auf das E-Modul der DiamantMetall-Verbundfolie

\subsection{Kornstruktur}

Die Oberflächen der betrachteten Proben aller Chargen stellen sich stark porös und zerklüftet dar. Die einzelnen

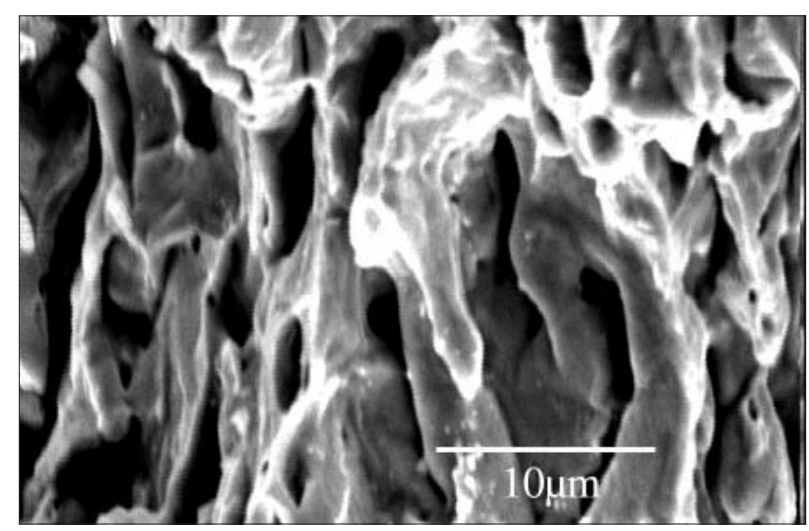

Abbildung 9: REM Aufnahme der Diamant-Metall-Verbundfolien mit Querbruch Probe D4; zu erkennen sind die vernetzten Dendriten
Kupferdendriten sind durch Walz- und Sinterprozesse miteinander vernetzt (Abb. 9). Ein klarer Unterschied der Kornstruktur zwischen den Chargen in Abhängigkeit von Zinn- und Diamantanteil sowie durch die Nachbehandlung Sintern und Nachwalzen konnte nicht nachgewiesen werden.

\subsection{Elastisches und plastisches Verhalten}

Möchte man Erkenntnisse über das plastische Verhalten eines Materials gewinnen, sind Eindringversuche mit einem Prüfkörper ein Mittel der Wahl. Bei der Nanoindentation können zusätzlich noch Aussagen zum elastischen Verhalten des Materials gemacht werden. Die Nanohärte und das reduzierte E-Modul wurden für verschiedene Proben bestimmt und sind in Abbildung 10 zusammengetragen.

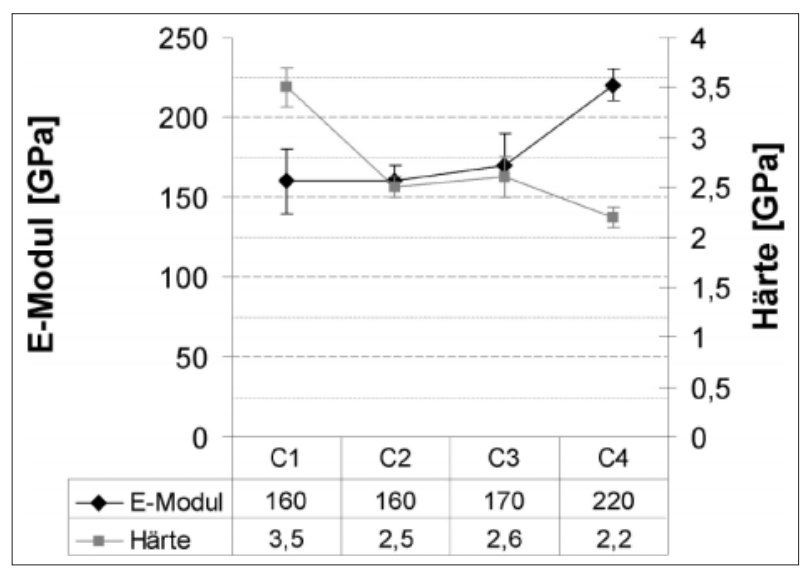

Abb. 10: Härte- und E-Modulvergleich der ersten vier Chargen der DiamantMetall-Verbundfolien

Die E-Modulwerte der Proben liegen mit ca.160 GPa dicht beieinander, nur Charge 4 liegt mit 220 GPa deutlich höher. Bei den Härtewerten der Chargen sticht Charge 1 mit 3,5 GPa hervor und liegt damit gut ein Drittel höher als die der Chargen 2, 3 oder 4. Diese Werte lassen keinen eindeutigen Rückschluss auf den Einfluss der Herstellungsparameter zu. Besonders die erwartete höhere Festigkeit der Bronzen C2 und C4 durch einen erhöhten Zinnanteil, konnte nicht belegt werden. Dies kann an der großen Oberflächenrauheit der Proben sowie an deren Porosität liegen.

\subsection{Tribologisches Verhalten}

Tribologische Laborversuche unter definierten Verhältnissen werden durchgefuihrt, um eine Abschätzung der Einsatzmöglichkeiten von Werkstoffen, z. B. in der Schleiftechnik, vorzunehmen. [9] Sie ermöglichen den Vergleich verschiedener Materialien bezuiglich Abtragsraten und Verschleißfestigkeit. Das tribologische Verhalten der Schleiffolien wurde in Zusammenarbeit mit der Fachgruppe VIII.1 „Tribologie und Verschleißschutz“ der Bundesanstalt für Materialforschung und -prüfung in Berlin (www.bam.de) untersucht. In einem Standardtestverfahren des reversierenden Gleitens wird eine Aluminiumoxid-Kugel mit einer konstanten Last von hier $10 \mathrm{~N}$ über den Verbundwerkstoff in einer linearen Bewegung hin und her gefahren. [9] Dabei tritt Verschleiß an der 
Kugel sowie auch an dem zu untersuchenden Material auf. Während des Versuches werden die Reibungszahl und der lineare Gesamtverschleiß erfasst. Die Verschleißspuren an der Kugel und an der Schleiffolie werden nach Versuchende lichtmikroskopisch und profilometrisch bestimmt. Trägt man den Gesamtverschleiß pro Zyklus doppelt logarithmisch gegen die Anzahl der Zyklen auf, ergibt sich ein Verlauf, wie in Abbildung 11 dargestellt. Es wurden Versuche über 5.000 (5k) und 50.000 (50k) Zyklen mit den Proben C5 und C6 durchgefuihrt. Beide Versuche uiber 5.000 Zyklen fallen in der logarithmischen Darstellung fast linear, was einem exponentiellen Abfall des Gesamtabtrages pro Zyklus entspricht. Dies bedeutet nicht zwangsläufig, dass die Schleifeigenschaften des Verbundwerkstoffes während des Versuches immer schlechter werden, sondern hängt vielmehr mit der Kugelform des Probekörpers zusammen. Die Kontaktfläche zwischen Kugel und Schleiffolie wächst mit steigendem Abtrag überproportional an. [10] Bei den Versuchen über 50.000 Zyklen fällt ein veränderter Verlauf der Graphen zwischen 10.000 und 20.000 Zyklen ins Auge. Die Funktionen beider Proben laufen dort fuir eine Zeit fast parallel zur Abszisse. Die Abtragsrate pro Zyklus liegt in diesem Bereich annähernd bei konstanten $2 \mathrm{~nm}$. Dieser Effekt hält für ein paar tausend Zyklen an, danach fallen die Graphen mit annähernd gleichen Anstieg wie in den ersten 10.000 Zyklen. Was diesen Effekt verursacht hat, ist bisher ungeklärt. Die Funktionen haben sehr ähnliche Anstiege und weisen das gleiche unregelmäßige Verhalten bei 10.000 Zyklen auf. Die unregelmäßigen Verschiebungen in y-Richtung sind durch die hohen Rauigkeiten der Proben und der damit verbundenen problematischen Abtragsmessung fuir die ersten Mikrometer verursacht. Aus den Funktionsverläufen des linearen Gesamtabtrages in Abbildung 11 können also keine klaren Unterschiede zwischen den Chargen festgestellt werden.

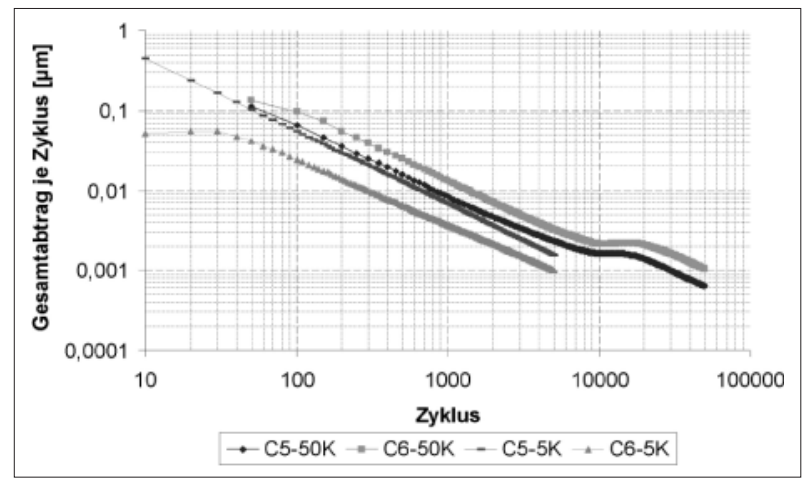

Abb. 11: Tribologisches Verhalten der Diamant-Metall-Verbundfolien: doppelt logarithmische Darstellung des gesamten Materialabtrages je Zyklus

Im Folgenden wurde dieser Gesamtabtrag des Abtrages differenzierter betrachtet und in den Verschleiß an der Schleiffolie und den Verschleiß an der AluminiumoxidProbe aufgeteilt. Die Verschleißspuren auf den Folien wurden profilometrisch vermessen und sind in den Abbildungen 12 und 13 dargestellt. Beide Bilder zeigen jeweils Tastschnitte der Versuche über 5.000 Zyklen (helleres oberes Profil), über 50.000 Zyklen (dunkles unteres Profil) und den für die Breite der Verschleißspur zutreffenden Kugelschnitt. Die Verschleißspuren von der Probe C5 sind deutlich flacher als die von C6. Daraus kann geschlos- sen werden, dass C5 über eine höhere Abriebfestigkeit verfügt. Diese ist höchstwahrscheinlich der höheren Diamantkonzentration zuzurechnen.

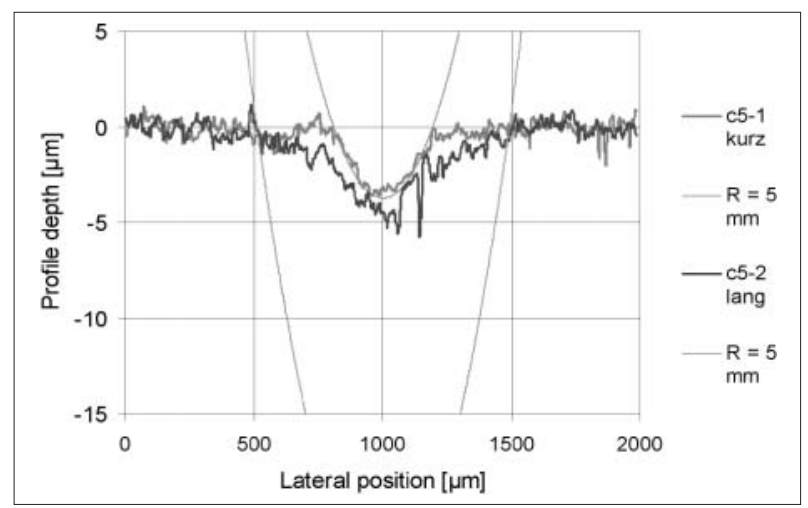

Abb. 12: Tastprofil der Verschleißspur der Diamant-Metall-Verbundfolie Probe C5

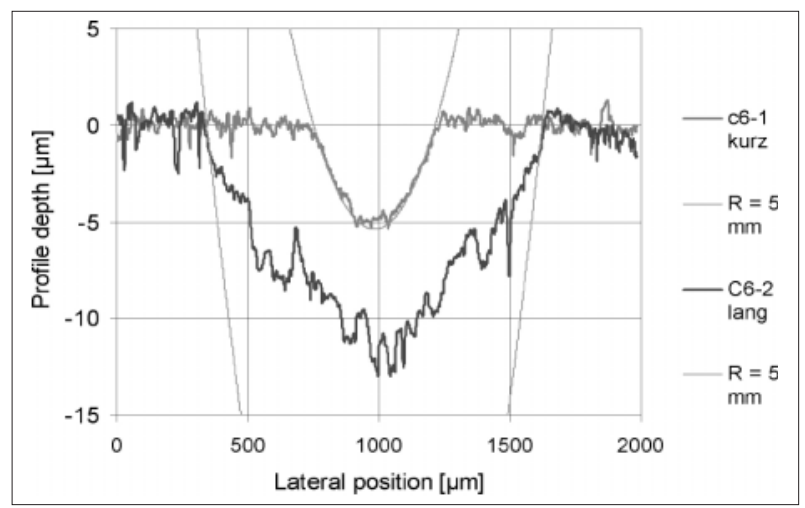

Abb. 13: Tastprofil der Verschleißspur in der Diamant-Metall-Verbundfolie Probe C6

Auffällig ist auch, dass fuir beide Proben der Tastschnitt nach 5.000 Zyklen fast exakt mit dem Kugelschnitt übereinstimmt. Dies bedeutet, dass in den ersten $3 \mu \mathrm{m}$ beziehungsweise $5 \mu \mathrm{m}$ Profiltiefe ausschließlich Material von der Schleiffolie und nicht von dem Probekörper abgetragen wurde. Da die Oberflächenrauheit der Proben einige Mikrometer beträgt, handelt es sich bei dem abgetragenen Material hauptsächlich um die Spitzen des Oberflächenprofils.

\section{Ausblick}

In der letzten Phase des Projektes sollen die bereits vorgestellten tribologischen Untersuchungen wiederholt und auf alle Chargen ausgedehnt werden, um Qualitätsmerkmale fuir die neuartigen Diamant-Metall-Verbundfolien aufzustellen. Diese Untersuchungen werden durch die praktische Anwendung des Produktes ergänzt. Erste Schleifversuche bei der Firma Optikkomponenten $\&$ Kristalle $\mathrm{GmbH}$, Berlin-Adlershof, haben vielversprechende Resultate in der Saphirbearbeitung ergeben. Die geschliffenen Saphirproben zeichneten sich durch eine hohe Planizität und eine geringe Oberflächenrauigkeit aus. Diese Schleifversuche muissen weiter geführt und die Resultate quantitativ erfasst werden. Es laufen weiterhin Vorbereitungen, diese Diamant-Metall-Verbundfolien mit kleineren Diamantkörnungen auch für den Polierprozess zu testen. 


\section{Danksagungen}

Die Forschungsarbeit wurde durch die „Arbeitsgemeinschaft industrieller Forschungsvereinigungen“ (AiF) im Programm „Innovationskompetenz mittelständischer Unternehmen“ (ProInno KF 0132808 KUK2) gefördert und finanziert. Marcel Muick und Michael Gruner danken ProInno für die finanzielle Unterstuitzung.

Das Projekt wird in Kooperation mit der Vollstädt Diamant $\mathrm{GmbH}$ durchgefuihrt, bei der wir uns für die engagierte Zusammenarbeit bedanken.

Wir danken Frau Carola Kubica und Herrn Dipl.-Krist. Ronald Ries von der TFH-Wildau für die tatkräftige Unterstuitzung bei der Probenpräparation und -analyse sowie fuir hilfreiche wissenschaftliche Diskussionen.

Alle Tribologieuntersuchungen wurden an der „Bundesanstalt für Materialforschung und -prüfung“ (BAM) in der Fachgruppe VIII.1 „Tribologie und Verschleißschutz“ durchgeführt. Wir danken besonders Prof. Dr. Erich Santner, Dr.-Ing. Dieter Klaffke und Dipl.-Ing. Manfred Hartelt für die schnelle, freundliche und kompetente Unterstuitzung.

\section{Literatur}

[1] www.net-lexikon.de/Korund.html, 07.05.2004.

[2] P. Dennis: Wirtschaftliches Doppelseitenplanschleifen mit CBN und Diamant Feinschleifscheiben; Diamond Buiseness, 2/2003.

[3] N. V. Novikov: Synthetische Superharte Materialien; Naukova dumka Kiev 1986.

[4] B. Ilschner, R. F. Singer; Werkstoffwissenschaften und Fertigungstechnik, Springer Verlag, 3. Auflage 2002.

[5] A. C. Fischer-Cripps: Nanoindentation; Springer-Verlag New York, 2002.

[6] A. Richter, R. Ries, R. Smith, M. Henkel and B. Wolf: Diamond and Related Materials 9 (2000) 170-184.

[7] M. Mück, A. Soshnikov, B. Wolf und A. Richter: Wissenschaftliche Beiträge der TFH Wildau (2003) 59-64.

[8] B. Wolf and A. Richter, New Journal of Physics 5 (2003) 15.1-15.17.

[9] D. Klaffke, F. Koesling, M. Hartelt; Tribologische Charakterisierung keramischer Werkstoffe mit reversierender Gleitbewegung; Tribologie + Schmierungstechnik, 48 . Jahrgang, 4/2001.

[10] J. Hertz: Reine und angewandte Mathematik 92 (1882) 156.

\section{Autoren}

Dipl.-Ing. (FH) Michael Gruner

Technische Fachhochschule Wildau

Fachbereich Ingenieur-/Wirtschaftsingenieurwesen Labor für Oberflächentechnik

Tel. +493375 508-217

E-Mail: mgruner@igw.tfh-wildau.de

Dipl.-Ing. (FH) Marcel Müick

Technische Fachhochschule Wildau

Fachbereich Ingenieur-/Wirtschaftsingenieurwesen Labor für Oberflächentechnik

Tel. +493375 508-286

E-Mail: mmueck@igw.tfh-wildau.de

\section{Prof. Dr. Asta Richter}

Technische Fachhochschule Wildau

Fachbereich Ingenieur-/Wirtschaftsingenieurwesen Labor für Oberflächentechnik

Tel. +493375 508-219

E-Mail: richter@pt.tfh-wildau.de 